\title{
Development of an Operation Guidance System to Reduce Pushing Load of Coke Ovens Based on Probabilistic Optimization
}

\author{
Yoshinari HASHIMOTO, ${ }^{1) *}$ Kazuro TSUDA, ${ }^{1)}$ Seitaro AKIYAMA ${ }^{21}$ and Michio HONMA ${ }^{21}$ \\ 1) Instrument and Control Engineering Research Department, Steel Research Laboratory, JFE Steel Corp., Minamiwatarida-cho, \\ Kawasaki, Kanagawa, 210-0855 Japan. \\ 2) Ironmaking Department, Kurashiki works, JFE Steel Corp., Kawasakidori, Mizushima, Kurashiki, Okayama, $712-8511$ Japan.
}

(Received on May 21, 2014; accepted on July 11, 2014)

\begin{abstract}
In recent years, the increase in the pushing load of coke ovens caused by aging has become a problem in steel works. In order to reduce this problem, an operation guidance system by a statistical approach was developed. The prediction model was developed based on an individual oven database, to deal with the variations in the characteristics of the ovens. The pushing process which involves complicated motion of coke grains can be regarded as a probabilistic phenomenon. Therefore, a guidance system using probabilistic optimization was constructed. The system was evaluated in actual operation, and the effect of reducing high pushing load ratio to $1 / 3$ has been confirmed.
\end{abstract}

KEY WORDS: pushing clogging; probabilistic optimization; locally weighted regression.

\section{Introduction}

In recent years, the age of coke ovens of steelmakers has exceeded 40 years, and problems associated with aging have become remarkable. When a coke-cake is pushed out of the oven, it is occasionally caught in the damaged wall. ${ }^{1)}$ This problem, which is called pushing clogging, leads to a significant decrease of production, because of the temperature drop during the downtime. Moreover, high pushing load due to the wall roughness damages the oven wall, resulting in the shortening of oven's lifespan. ${ }^{2)}$ To reduce these problems, steelmakers take countermeasures, such as changing the blend ratio of coals, ${ }^{3)}$ speeding up the wall repair, and so on. In this research, we developed an operation guidance system to reduce the pushing load.

The operation cycle of a coke oven consists of loading, coking and pushing, and the coking time is about 16-20 hours. Operators make pushing plans for roughly 40 ovens, before starting their 8 hour shifts. In the present operation, the push timing is determined by a carbonization determination system which is based on the information of the temperature trends of the coke oven gas. However, operators sometimes modify the timing in response to visual information obtained by observation through loading holes, because the system cannot refer the information of the wall condition and the distribution of fire extinguishing degree. Consequently, personal judgment depending on the ability of the operators is inevitable, which causes variations in operation.

To improve the operation, we proposed an operation guidance system based on a database by a statistical approach. We constructed a logic to predict the pushing load, and to calculate

* Corresponding author: E-mail: y-hashimoto@jfe-steel.co.jp DOI: http://dx.doi.org/10.2355/isijinternational.54.2623 optimal timing to avoid high pushing load. The phenomenon of pushing the coke cake, which involves transformation of the cake, is complicated. ${ }^{1,4,5)}$ As the pushing load is affected by subtle differences in coke grain arrangement and the wall conditions, we regarded it as a probabilistic variable, and developed a guidance system based on probabilistic optimization.

In this paper, the procedure for predicting the pushing load is presented in section 2. The detail of guidance system based on probabilistic optimization is described in section 3 . Implementation of the system and its evaluation in actual operation are presented in section 4. Finally, we conclude this paper in section 5 .

\section{Prediction of the Pushing Load}

In this section, the procedure for predicting pushing load is explained. Pushing load is governed by many factors as shown in Fig. 1. The clearance means the gap between the coke cake and the wall which affects the friction between them. ${ }^{6}$ ) This can be changed by the blend ratio of coal, and other operating condition, such as coking time and flue temperature. The stability of the coke cake when it is pushed out of the oven is

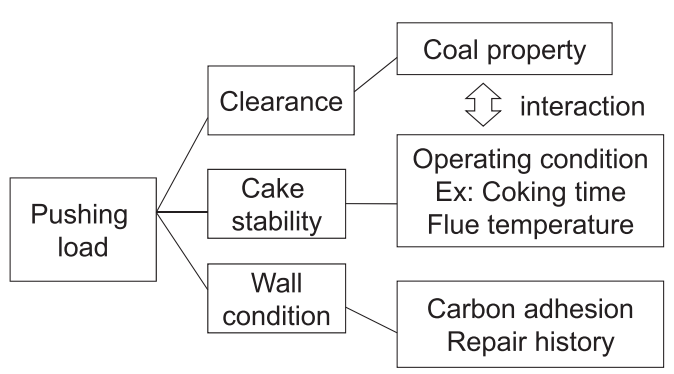

Fig. 1. Explanatory variables of pushing load. 


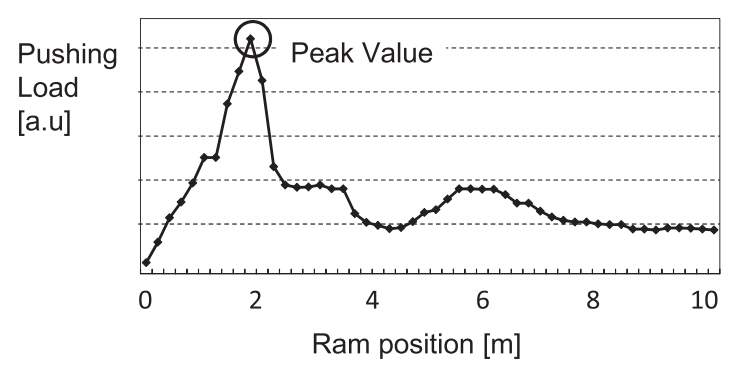

Fig. 2. Peak value of pushing load.

crucial to keep the clearance during the process. The wall condition can be evaluated by the degree of wall roughness. ${ }^{7,8)}$

In this study, critical factors available from the online process computer were selected, which are coking time, coal amount, furnace temperature, and water ratio. Furthermore, actual load in the previous push timing was added to the explanatory variables in order to adapt to the change of wall condition and the coal blend ratio, which cannot be referenced online. The pushing load was predicted from these explanatory variables.

As an index of the pushing load, we utilized the peak value of the pushing load during the pushing process as shown in Fig. 2. In this study, we focused on a certain type of pushing clogging, where the peak position is less than $5 \mathrm{~m}$ from the oven door. The peak of the pushing load in this range is relatively predictable, because the coke cake maintains its shape and the clearance is determined mainly by the operating condition. Therefore, the peak value before the ram reaches $5 \mathrm{~m}$ was defined as the objective variable.

In old coke ovens, the influence of operational conditions on the pushing load greatly depends on the characteristics of each oven, because of the variations in the wall profile. Therefore, the operation database was separated for each oven, and the prediction was conducted based on the individual oven data.

The concept of locally weighted regression ${ }^{9-11)}$ (LWR) was applied when calculating the prediction value. This method forms a regression equation, weighing each datum by a degree of similarity with target data point, and makes it possible to maintain prediction accuracy in cases where the data distribution is nonlinear. The calculation is presented in Eqs. (1)-(5), where $\mathbf{X}$ is the explanatory data matrix, $p$ is the number of explanatory variables, $N$ is the number of data, $\mathbf{y}$ is the objective variable data, $\Omega$ is the weight matrix, vector $\mathbf{a}$ is regression coefficient, $\mathbf{x}_{\text {query }}$ is the target operational condition, and $y_{\text {pre }}$ is the predicted pushing load, respectively. The suffix $i$ corresponds to $i$ th datum.

$$
\begin{aligned}
& \mathrm{X}=\left(\begin{array}{cccccc}
x_{11} & x_{12} & \cdot & \cdot & x_{1 p} & 1 \\
x_{21} & \cdot & \cdot & & x_{2 p} & 1 \\
\cdot & & & & & \\
\cdot & & & & & \\
x_{N 1} & \cdot & \cdot & \cdot & x_{N p} & 1
\end{array}\right) \text {................. }
\end{aligned}
$$

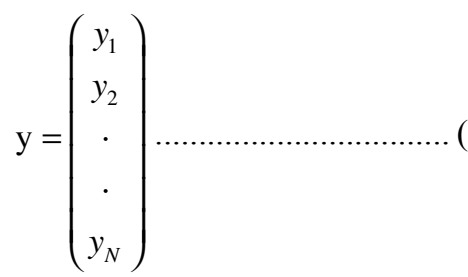

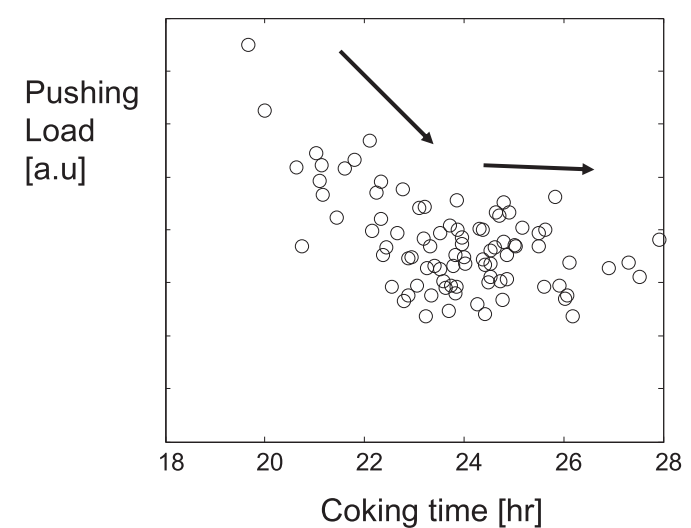

Fig. 3. Nonlinear relationship between coking time and pushing load.

$$
\begin{gathered}
\Omega=\operatorname{diag}\left(w_{1}, w_{2}, w_{3}, \cdots, w_{N}\right) \\
\mathbf{a}=\left(\mathbf{X}^{T} \boldsymbol{\Omega X}\right)^{-1}\left(\mathbf{X}^{T} \Omega \mathbf{y}\right) \ldots \\
y_{\text {pre }}=\left(\mathbf{x}_{\text {query }} 1\right) \cdot \mathbf{a} \ldots \ldots . .
\end{gathered}
$$

The weight of each datum is presented in Eq. (6), where $G C T$ is the coking time and date is the sampling date of each datum. The suffix $i$ corresponds to $i$ th data, and suffix query means the target condition. $G$ and $F$ are adjustable parameters. First, the weight of coking time was imposed because of the nonlinearity between coking time and pushing load as shown in Fig. 3. This figure suggests that the effect of reducing load by the shrinkage of coke cake is saturated when the coking time is long. Considering the degree of nonlinearity, the parameter $G$ was set to 2.5 [hours].

$w_{i}=\exp \left(\frac{-\left(G C T_{\text {query }}-G C T_{i}\right)^{2}}{G^{2}}\right) \cdot \exp \left(\frac{-\left(\text { date }_{\text {query }}-\text { date }_{i}\right)}{F}\right) \ldots$

In the case of normal LWR, the similarity is defined by using the data of all explanatory variables. However, if the relationship between an explanatory variable and the objective variable is linear, this kind of weight is not necessary, because normal linear regression works well. It is noteworthy that the weight should be imposed only on an explanatory variable which has a nonlinear relationship with the objective variable.

The other weight in Eq. (6) was multiplied to express a time difference between the target point and each datum. The condition of oven walls frequently changes due to wall maintenance. Therefore, the forgetting factor was imposed to put greater weight on recent data. Considering the frequency of the wall maintenance, the parameter $F$ was set to 100 [days].

The prediction of pushing load based on the weighted linear regression was conducted in this manner. The predicted value was plotted against the actual pushing load as shown in Fig. 4 (Right). The pushing load is normalized by the maximum load of the pusher's motor. The estimated load and the actual load were in good agreement. To confirm the effect of LWR, the result of normal linear regression is shown in Fig. 4 (Middle). The effect of improving prediction accuracy can be confirmed. For the sake of comparison, the result of the prediction without separating the data for each oven is shown in Fig. 4 (Left). The effect of individual oven database can be confirmed. 

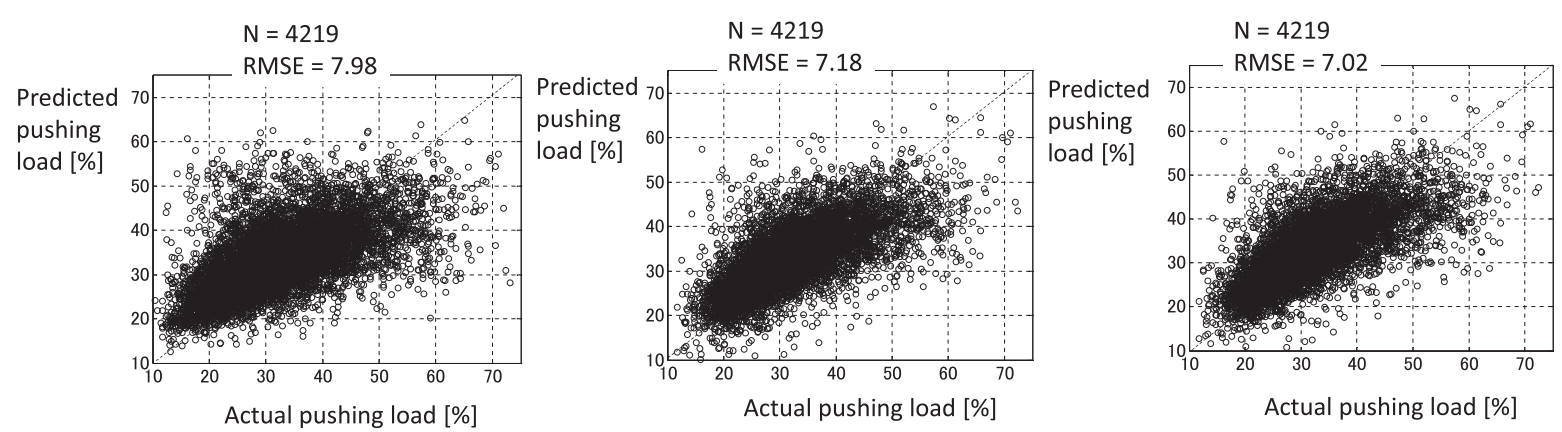

Fig. 4. Prediction accuracy of pushing load. (Left): Without individual oven database. (Middle): With individual oven database, without LWR. (Right): With individual oven database, with LWR.

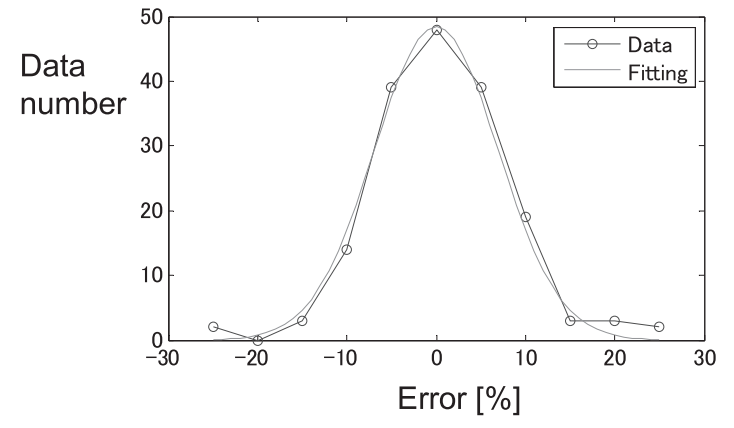

Fig. 5. Distribution of the prediction error.

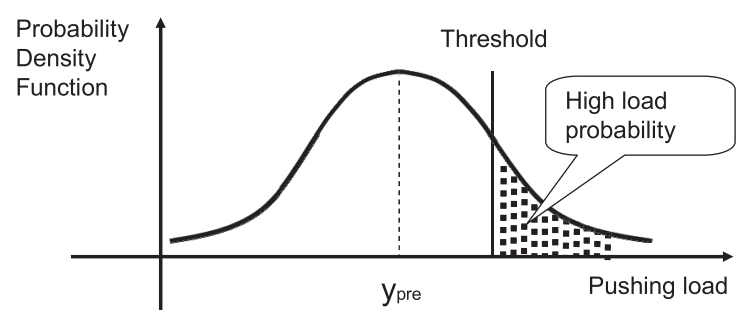

Fig. 6. Definition of the high load probability.

\section{Development of a Guidance System Based on Proba- bilistic Optimization}

As mentioned in section 1, the pushing load can be regarded as a probabilistic variable. Quantitative expression of the high load probability and optimization of the push timing based on the probability are presented in this section. $^{12)}$

First, the residual distribution of the local regression as mentioned in section 2 was investigated. As shown in Fig. $\mathbf{5}$, it can be approximated as a Gaussian distribution. Therefore, the probability density function of the pushing load can be expressed as the Gaussian distribution whose center value is the predicted value by the local regression.

Next, the high load probability was defined by integrating the probability density function above a threshold as described in Eq. (7), where $\sigma$ is the root mean square error of the prediction, prob(Load $>$ Th) is the high load probability, $y_{\text {pre }}$ is the predicted pushing load (Fig. 6). The probability density function is normalized by the center value $y_{\text {pre }}$ and standard deviation $\sigma$, and the area over the threshold is calculated. In this case, the threshold of the load was set to $50 \%$. The probability and actual frequency of high pushing load above the threshold are compared in Fig. 7, which shows good agreement between the two.

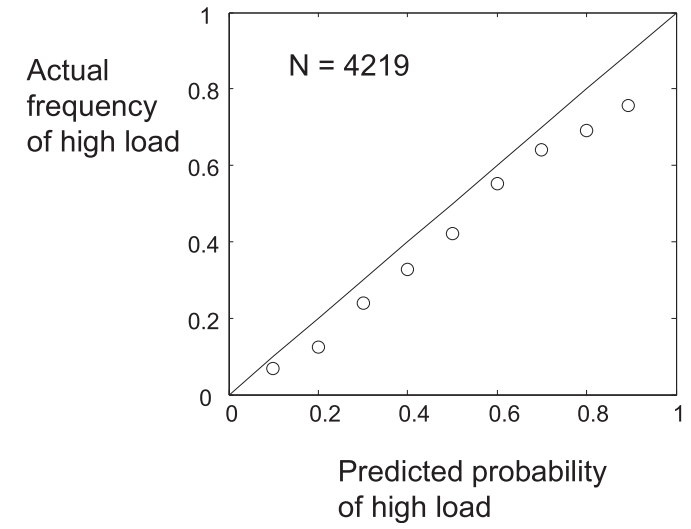

Fig. 7. Relationship between predicted high load probability and actual frequency of high push load above threshold.

$$
\begin{aligned}
& \operatorname{prob}(\text { Load }>T h)=f\left(\frac{T h-y_{\text {pre }}}{\sigma}\right) \\
& f(x)=\frac{1}{\sqrt{2 \pi}} \int_{x}^{+\infty} e^{-\frac{t^{2}}{2}} d t
\end{aligned}
$$

The optimal timing of pushing can be determined by using the high load probability. In general, the risk of high load can be reduced by increasing coking time as indicated in the relationship between the pushing load and the coking time in Fig. 3. However, redundant coking time decreases production. Operators usually determine the optimal timing by taking a balance between the two.

In this research, the cost function was designed following this idea as described in Eq. (9), where $A$ is the downtime caused by pushing clogging. In this case, $A$ was set to 24 hours. The optimal coking time (i.e. push timing) which minimizes the function was determined. An example of the optimization is shown in Fig. 8. In this way, the guidance logic based on the tradeoff between prolonging the coking time and the risk of pushing clogging was established.

$$
f\left(\mathbf{x}_{\text {query }}\right)=A \times \operatorname{prob}(\text { Load }>T h)+G C T_{\text {query }}
$$

\section{Evaluation of Guidance System in Actual Operation}

\subsection{Implementation of Guidance System}

The operation guidance system based on the probabilistic optimization was implemented in the coke factory at Kurashiki works in JFE Steel. The outline of the system is shown in Fig. 9. Operational data on the process computer are separated for individual oven, and the high load probability and 


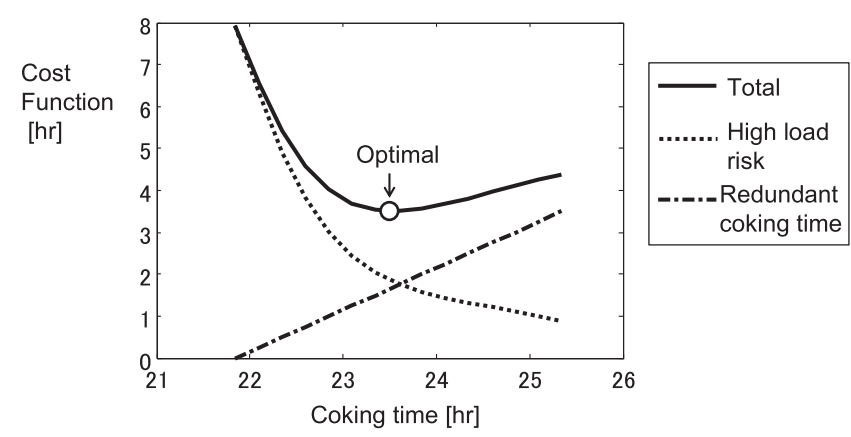

Fig. 8. Example of optimization of coking time.

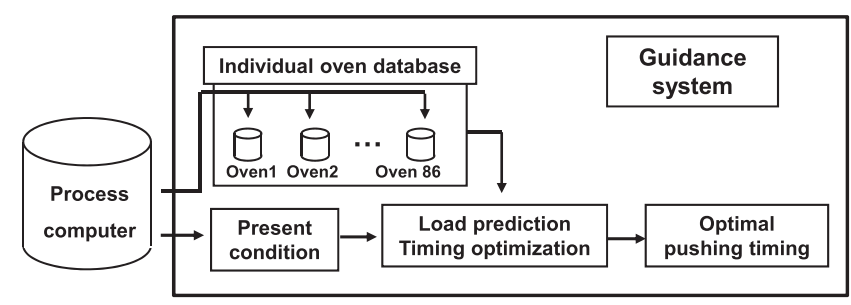

Fig. 9. Outline of guidance system.

the optimal push timing are calculated based on the present operational condition. The ovens of one shift are listed, and the information on the optimal pushing timing and the change of the high load probability are presented to operators. The guidance information is updated every 10 minutes, reflecting the newest data. The operators utilized the guidance system combining with the conventional carbonization system.

\subsection{Effect of Reducing Pushing Load by the Guidance System}

The frequency of high pushing load was compared between the case where the guidance was followed by the operators and the case where the operators pushed earlier than the recommended timing by more than one hour. As shown in Table 1, the frequency of high load increased by 3 times when the guidance was not followed. Therefore, the high load frequency can be reduced to $1 / 3$ by following the guidance. This survey was also conducted for each operation shift as shown in Table 2. It was found that the guidance system was effective in every case. The pushing load took high value occasionally although the guidance was followed. In such cases, the cause of the high load was not the shortage of coking time, but other factors such as the wall roughness or the instability of the coke cake.

There was a tendency that the operators pushed the coke cake earlier than the guidance when the carbonization system indicated the fire extinguishing, which often resulted in high pushing load. The clearance between the coke cake and oven wall might not be enough for pushing, even when the carbonization seemed to finish judging from the information of temperature trends of coke oven gas. In old coke ovens, the pushing load may be governed by the clearance at certain part of the oven wall, the information of which does not appear clearly in the temperature of coke oven gas. The clearance could have been increased by putting off the pushing timing as the guidance system recommended. Especially, in the case of the oven in which the relationship between the coking time and the pushing load is clear, the guidance system is quite reliable.
Table 1. Effectiveness of guidance system (overall).

\begin{tabular}{c|ccc}
\hline Lush timing & Below threshold & Above threshold & High load ratio \\
\hline Guidance followed & $\mathrm{N}=2713$ & $\mathrm{~N}=39$ & $1.4 \%$ \\
Not followed & $\mathrm{N}=980$ & $\mathrm{~N}=48$ & $4.7 \%$ \\
\hline
\end{tabular}

Table 2. Effectiveness of guidance system (Each operation team).

Team A

\begin{tabular}{l|ccc}
\hline Lush timing & Below threshold & Above threshold & High load ratio \\
\hline Guidance followed & $\mathrm{N}=598$ & $\mathrm{~N}=11$ & $1.8 \%$ \\
Not followed & $\mathrm{N}=253$ & $\mathrm{~N}=16$ & $5.9 \%$ \\
\hline
\end{tabular}

Team B

\begin{tabular}{l|ccc}
\hline Lush timing & Below threshold & Above threshold & High load ratio \\
\hline Guidance followed & $\mathrm{N}=672$ & $\mathrm{~N}=13$ & $1.9 \%$ \\
Not followed & $\mathrm{N}=236$ & $\mathrm{~N}=16$ & $6.3 \%$
\end{tabular}

Team C

\begin{tabular}{l|ccc}
\hline Lush timing & Below threshold & Above threshold & High load ratio \\
\hline Guidance followed & $\mathrm{N}=717$ & $\mathrm{~N}=9$ & $1.2 \%$ \\
Not followed & $\mathrm{N}=273$ & $\mathrm{~N}=7$ & $2.5 \%$ \\
\hline
\end{tabular}

Team D

\begin{tabular}{l|ccc}
\hline Lush timing & Below threshold & Above threshold & High load ratio \\
\hline Guidance followed & $\mathrm{N}=730$ & $\mathrm{~N}=6$ & $0.8 \%$ \\
Not followed & $\mathrm{N}=216$ & $\mathrm{~N}=9$ & $4.0 \%$ \\
\hline
\end{tabular}

\section{Conclusion}

In this research, we constructed a guidance system to reduce the pushing load, which was regarded as a probabilistic variable. The prediction model was developed based on individual oven database, to deal with the variations in the characteristic of the ovens. The optimal pushing timing was determined by the cost function which takes a balance between prolonging coking time and the risk of pushing clogging. The guidance system was evaluated in actual operation, and the effect of reducing high pushing load ratio to $1 / 3$ has been confirmed.

\section{REFERENCES}

1) S. Watakabe, K. Takeda, H. Suginobe and H. Itaya: Tetsu-to-Hagané, 84 (1998), 165.

2) I. Shimoyama, T. Yamamoto and K. Fukata: ISIJ Int., 50 (2010), 1048 .

3) S. Watakabe: CAMP-ISIJ, 10 (1997), 154.

4) S. Aizawa, K. Uebo, S. Yoshida and Y. Komai: CAMP-ISIJ, 18 (2005), 92.

5) K. Fukuda and T. Arima: CAMP-ISIJ, 10 (1997), 155.

6) K. Nushiro, T. Matsui, K. Hanaoka, S. Igawa and K. Sorimachi: Tetsu-to-Hagané, 81 (1995), 625.

7) M. Sugiura, T. Nakagawa, T. Arima, K. Kato, M. Sakaida, Y. Morizane, A. Sano and K. Irie: ISIJ Int., 53 (2013), 583.

8) T. Nakagawa, T. Arima, K. Kato and M. Ando: CAMP-ISIJ, 15 (2002), 766.

9) H. Shigemori, K. Nanbu, R. Nagao, Y. Araki, N. Mizushima, M. Kano and S. Hasebe: Trans. SICE, 46 (2010), 472.

10) H. Shigemori, M. Kano and S. Hasebe: J. Process Contr., 21 (2011), 293.

11) W. S. Cleveland and S. J. Devlin: J. Am. Stat. Assoc., 83 (1988), 596.

12) A. Charnes and W. W. Cooper: Oper. Res., 11 (1963), 18. 